\title{
Screening of Eggplant Germplasms against Phomopsis Blight and Fruit Rot Caused by Phomopsis Vexans
}

\author{
M.M. Islam ${ }^{1 *}$, M.I. Faruk ${ }^{2}$, M.S. Rahman ${ }^{3}$, K.E. Jahan ${ }^{4}$, M. Asaduzzaman ${ }^{5}$ \\ 1, 3, ${ }^{4}$ Senior Scientific Officer, Plant Pathology Division, BARI, Gazipur \\ ${ }^{2}$ Principal Scientific Officer, Plant Pathology Division, BARI, Gazipur \\ ${ }^{5}$ Senior Scientific Officer, Olericulture Division, HRC, BARI, Gazipur
}

*Corresponding Author: M.M. Islam, Senior Scientific Officer, Plant Pathology Division, BARI, Gazipur

\begin{abstract}
Eggplant (Solanum melongena L.) is one of the most important vegetable in Bangladesh. It is very popular and nutrient rich vegetable. Eggplant can be grown round the year in Bangladesh. A center of origin is believed to be in the region of Bangladesh and Myanmar (Indo-Burma border). But the production and yield of eggplant is very low in Bangladesh due to several diseases. homopsis blight and fruit rot disease is the most devastating one among the diseases. The experiment was conducted at the field of Plant Pathology Division, Bangladesh Agricultural Research Institute (BARI), Gazipur during the three consecutive cropping seasons 2016-17, 2017-18 and 2018-19 to identify the resistant germplasms/entries of eggplant against phomopsis blight and fruit rot disease causing Phomopsis vexans. Altogether 53 germplasms were collected from different eggplant pockets of the country, research Institute and abroad. Collected eggplant vars/entries showed differential reactions against Phomopsis vexans. Percent disease index (PDI) of fruit ranged from 0.0 -61.35\% among the 53 accessions where 13 cultivars were found highly susceptible, 21 cultivars susceptible, 8 cultivars moderately susceptible and 9 showed moderately resistant while cultivar Katabegun-WS and wild accession were resistant. Existence of variation in eggplant cultivars was envisaged. Relatedness of eggplant against phomopsis disease reaction could be potential information for developing the disease resistant cultivar of its.
\end{abstract}

Keywords: Eggplant, Screening, Phomopsis blight and fruit, Phomopsis vexans.

\section{INTRODUCTION}

Eggplant (Solanum melongena L.) is an important vegetable in Bangladesh cultivated round the year in all districts of the country (Anon. 1988). The total acreage of eggplant is 51,165 hectares with total annual production of 5,16,007 tones (Anon., 2019). Its position is second in vegetable crops in terms of production next to potato. This is a vegetable available throughout the year in the market and especially during the lean period. Eggplant is thus regarded as a cash crop. A large number of eggplant cultivars are grown in Bangladesh, which show a wide range of variations in yield performance. But, eggplant suffers from 12 diseases of which fruit rot caused by Phomopsis vexans (Sacc. and Syd) Harter is a devastating one. The organism of the disease Phomopsis vexans remains viable for about 14 months in soil debris and in the seed from infected fruits (Kalda et al., 1977). The pathogen is reported both externally and internally seed borne. The disease was first reported from Gujrat in 1914 and since then from many parts of India. Occurrence of the disease in Bangladesh has been reported by Fakir (1983) and Ahmad (1987). The disease has become a major constraint in intensive cultivation of eggplant. Crop losses due to this disease are evident, loss ranges from 15-20\% in general but 30-50\% in severe case (Das, 1998). It is a serious disease which may cause damping off symptoms if attacked at seedling stage. When the leaves are infected, small circular spots appear which become grey to brown with a light color centre. The infected leaves may turn yellow and die. Lesion may also develop on petiole and stem causing blighting of affected portions. In course of time, the spot enlarges and produces concentric circular area. Ultimately, the fruits become mummified and rotten (Kumar et al., 1986). There is no recognized resistant variety of eggplant against fruit rot disease till today. Our farmers are reluctant to buy fungicides. Conversely, they are now indulged to indiscriminate use of insecticides, causing environment pollution. Under this scenario, it appears that a single input would be the best suited approach for management of eggplant diseases and insect pest, 
it must be a basic component of eggplant production and obviously it is a resistant variety. Therefore, the present research work was undertaken to identify the resistant eggplant cultivar/cultivars against phomopsis blight and fruit rot disease causing Phomopsis vexans.

\section{Materials ANd Methods}

The seeds of 53 eggplant cultivars were collected from commercial farmers of major eggplant growing areas, local markets, Horticulture Research Center (HRC, BARI), renowned seed companies etc during August' 2016 to September' 2016 (Table 1). Seedlings were raised in plastic trays in the net house with proper care and management in the consecutive three years. Trays were prepared by mixing soil, sand and well decomposed cow dung in the proportion of 2:1:1. The prepared soil was heaped like a square block. Formalin solution (4\%) @ $200 \mathrm{ml} / \mathrm{cft}$ soil was mixed with the soil heap and the soil was covered by a polythene sheet for 48 hours. After 7 days, surface sterilized trays were filled up with the sterilized soil (Dhasgupta, 1988). Seeds were sown in a diametric line and labeled by a permanent marker. Watering was done to maintain the soil moisture. Shade was provided to save the young and delicate seedlings from heavy showering and scorching sunlight. A piece of medium high land with well drainage system was selected and prepared by ploughing, followed by laddering. The soil was well pulverized for tilth condition. Weeds and stubbles were removed. During field preparation, fertilizers and manures were applied at recommended doses (Anon. 2012). Seedlings of age 25 days were transplanted in the field followed by watering. Fifteen seedlings were planted in each subplot maintaining plant-to-plant distance $75 \mathrm{~cm}$ and line-to-line $1 \mathrm{~m}$. Inoculation of 5 plants in each plot of each cultivar/entry was done at flowering stage and 5 plants at fruiting stage. Seventy milliliter spore suspension $\left(5 \times 10^{6}\right.$ spore/ml) sprayed for each plant (Khan, 1999). Another 5 plants were kept uninoculated (control). For ensuring better infection, the spraying was done at afternoon and inoculated plants were covered with moist transparent polythene sheet for 24 hours. Data were recorded on \% Leaf infection, \% LAD (Leaf Area Diseased), \% Flower infection, \% Fruit infection and \%FAD (Fruit Area Diseased).Percent LAD (Leaf Area Diseased) and FAD (Fruit Area Diseased) were measured by eye estimation. Area of a single leaf / fruit was considered as $100 \%$. Deducting the healthy area, the diseased area was estimated. Average of \% LAD / FAD was then calculated dividing the total number of investigated leaves/fruits (Islam, 2005). After inoculation, records on expression of symptom on leaf, flower and fruit were taken at an interval of seven days. Infection was expressed in percentage. The test entries were placed in various categories of resistance and susceptibility utilizing the standard area diagram of Islam et al. (1990) with slight modification.

Table1. Name and sources of collected germplasms of eggplant

\begin{tabular}{|l|l|l|l|}
\hline Lot & Cultivar /germplasm & Source of collection & Collector \\
\hline 1 & Zhumki & Nandina, Jamalpur & M. Islam \\
\hline 2 & ISD-006 & BARI, Joydebpur, Gazipur & M. Islam \\
\hline 3 & Laffa & Marichar char, Mymensingh & M. Islam \\
\hline 4 & Laffa & Gaffargaon, Mymensingh & M. Islam \\
\hline 5 & Laffa W & Rupgonj, Narayangonj & R. Islam \\
\hline 6 & Laffa B & Rupgonj, Narayangonj & R. Islam \\
\hline 7 & Laffa S & Sherpur & Y. Ali \\
\hline 8 & Laffa & BAU, Mymensingh & M. Islam \\
\hline 9 & Volanath & Marichar char, Mymensingh & M. Islam \\
\hline 10 & Thamba & Marichar char, Mymensingh & M. Islam \\
\hline 11 & Dohazari R & Dohazari, Chittagong & R. Islam \\
\hline 12 & Dohazari G & Dohazari, Chittagong & R. Rashed \\
\hline 13 & Borka & Nuton Bazar, Mymensingh & B. Meah \\
\hline 14 & Khatkhatia & Bhurungamari, Kurigram & I. Islam \\
\hline 15 & Khatkhatia BAU & BAU, Mymensingh & M. Islam \\
\hline 16 & Kaikka & Nandina, Jamalpur & Y. Ali \\
\hline 17 & Kaikka & Gaffargaon, Mymensingh & M. Islam \\
\hline 18 & Islampuri BADC & BADC, Mymensingh & R. Islam \\
\hline 19 & Jashore & Monirampur, Jashore & Howlader \\
\hline 20 & Dharala & Betila, Manikgonj & Rahman \\
\hline 21 & Nayantara & BARI, Gazipur & M. Islam \\
\hline 22 & Uttara & BARI, Gazipur & M. Islam \\
\hline 23 & Kazla & BARI, Gazipur & M. Islam \\
\hline
\end{tabular}




\begin{tabular}{|l|l|l|l|}
\hline 24 & Singnath & Chandina, Comilla & R. Islam \\
\hline 25 & BL -118 & BARI, Gazipur & M. Islam \\
\hline 26 & Dundhul & Gaffargaon, Mymensingh & M. Islam \\
\hline 27 & Botli begun & Marichar char, Mymensingh & M. Islam \\
\hline 28 & Marichbegun S & Sherpur & Y. Ali \\
\hline 29 & Marich begun E & Sherpur & Y. Ali \\
\hline 30 & Marich begun L & Sherpur & Y. Ali \\
\hline 31 & Katabegun WS & Paba, Rajshahi & M. Islam \\
\hline 32 & Bijoy & Modhukhali, Faridpur & R. Islam \\
\hline 33 & Amjuri, & Mymensingh & M. Islam \\
\hline 34 & EG-190 & AVRDC, Taiwan & M.Uddin \\
\hline
\end{tabular}

Table1. (contd.)

\begin{tabular}{|l|l|l|l|}
\hline Lot & Cultivar /germplasm & Source of collection & Collector \\
\hline 35 & China oblong & Paba, Rajshahi & Y. Ali \\
\hline 36 & Wild & BAU, Mymensingh & M. Islam \\
\hline 37 & Rupgonj - L & Rupgonj, Narayangonj & R. Islam \\
\hline 38 & Ishurdi - WS & Ishurdi, Pabna & Y. Ali \\
\hline 39 & Ishurdi - BS & Ishurdi, Pabna & Y. Ali \\
\hline 40 & Putabegun & Chittagong & N. Uddin \\
\hline 41 & Longla long & Longla, Moulivibazar & M. Islam \\
\hline 42 & Shingnath - S & BAU, Mymensingh & M. Islam \\
\hline 43 & Longla talbegun & Longla, Moulivibazar & M. Islam \\
\hline 44 & Islampuri & Islampur, Jamlpur & M. Islam \\
\hline 45 & Thapra & Gabtoli, Bogra & M. Islam \\
\hline 46 & Mireshawry & Mireshawry, Chittagong & M. Islam \\
\hline 47 & Menter & Gabtoli, Bogra & M. Islam \\
\hline 48 & Salta & Burirhat, Rangpur & M. Islam \\
\hline 49 & Iribegun & Betila, Manikgonj & M. Islam \\
\hline 50 & Eye-red & Betila, Manikgonj & M. Islam \\
\hline 51 & Khatkhatia & Khatkhatia, Rangpur & M. Islam \\
\hline 52 & Deembegun & BAU, Mymensingh & M. Islam \\
\hline 53 & Comilla L & Comilla & M. Islam \\
\hline
\end{tabular}

\section{RESUltS}

Pooled data for the years 2016-2017, 2017-2018 and 2018-2019 on the incidence and severity of Phomopsis blight and fruit rot on eggplant cultivars are given in table 2.The highest leaf infection and PDI - leaf was recorded in Islampuri I $(62.21 \%, 31.19 \%)$ and Rupgonj L $(61.74 \%, 30.85 \%)$. The lowest leaf infection and PDI (leaf) was observed in Ishurdi WS $(8.25 \%, 4.15 \%)$ preceded by Ishurdi BS $(8.40 \%, 7.12 \%)$, Marichbegun S $(8.42 \%, 4.22 \%)$, Marichbegun E $(8.76 \%, 4.31 \%)$, Marichbegun L $(9.08 \%, 4.53 \%)$, ISD-006 $(10.47 \%, 4.49 \%)$, Dhundul $(11.90 \%, 6.01 \%)$, Jashore $1(13.68 \%, 6.88 \%)$ and Laffa M $(13.99 \%, 7.02 \%)$. Katabegun WS and wild accession had no leaf infection. In case of flower infection, the cultivars varied significantly among themselves. The highest flower infections were observed in cv. Kazla, Menter and Zhumki. Higher scale of flower infection 35 to $40 \%$ were found in cvs. Laffa B, Khatkhatia R, EG-190, Uttara, Rupgonj L, Islampuri I, Borka, China and Eyered. Katabegun WS and wild accession had no flower infection. Three cultivars viz. Ishurdi WS, Ishurdi BS and Marichbegun S had lower flower infection $(<10 \%)$. Rest of the cultivars had flower infection ranged from $12.45 \%$ to $36.43 \%$ (Table 2). Trend of fruit infection and PDI (fruit) was more or less similar to that of leaf and flower infection of the different accessions with some deviation. The highest fruit infection was observed in cultivar Uttara (71.36\%) which was statistically identical with the cultivar Kazla (69.87\%) followed by EG-190, Menter, Islampuri I, Laffa B, Uttara, Boruka, China, Islampuri-BADC, Laffa W, Longla T, Longla L, Putabegun and Eyered. Katabegun WS and wild accession had no fruit infection. One cultivar viz. Ishurdi WS had less than $10 \%$ and six cultivars namely ISD-006, Jashore L, Dhundul, Marichbegun S, Ishurdi BS and Salta had less than $20 \%$ fruit infection. Rest of the cultivars had fruit infection ranged from $21.38 \%$ to $43.33 \%$ (Table 2).

Percent Disease Index (PDI-fruit) varied significantly among the cultivars. The higher grade of PDI (fruit) was recorded in cultivars Zhumki, Kazla, Eg-190, Menter, Rupgonj L, Islampuri I, Laffa B, Borka, Uttara, China, Laffa W, Islampuri-BADC, Longla $\mathrm{T}$ and Longla and those were graded as highly susceptible (HS). Cultivar Katabegun WS and wild accession had no PDI (fruit) as they had no 
fruit infection and graded as resistant (R). The lowest PDI (fruit) was observed in cultivar Ishurdi WS $(6.66 \%)$ that was statistically identical to Jashore L $(8.55 \%)$ and Ishurdi BS $(8.10 \%)$ and those were graded as moderately resistant (MR). The cultivars Laffa M, Thamba, Dohazari R, Kaikka N, Singnath C, Amzuri, Iribegun and Deem begun had 19.04 to $24.98 \%$ PDI (fruit) and were graded as moderately susceptible (MS). The PDI (fruit) ranged from $22.42-42.32 \%$ in the rest of the cultivars and were graded as susceptible (S).Considered the incidence and severity of Phomopsis blight and fruit rot of eggplant pooled for the years 2016-2017, 2017-2018 and 2018-2019, only one cultivar Katabegun WS and the wild accession showed resistant reaction (R) against the disease. While 9 others showed moderately resistant (MR), 8 showed moderately susceptible (MS), 21 susceptible (S) and 13 showed highly susceptible (HS) reactions (Table 2).

Table2. Disease reaction of eggplant germplasms/cultivars against Phomopsis vexans causing Phomopsis blight and fruit rot of eggplant (Pooled for the years of 2016-17, 2017-18 and 2018-19).

\begin{tabular}{|c|c|c|c|c|c|c|c|}
\hline Sl. No & $\begin{array}{c}\text { Germplasms } \\
\text { /cultivars }\end{array}$ & $\begin{array}{l}\text { \% Leaf } \\
\text { infection }\end{array}$ & $\begin{array}{l}\text { PDI } \\
\text { (Leaf) }\end{array}$ & $\begin{array}{l}\text { \% Flower } \\
\text { infection }\end{array}$ & $\begin{array}{l}\text { \% Fruit } \\
\text { infection }\end{array}$ & $\begin{array}{l}\text { PDI } \\
\text { (fruit) }\end{array}$ & $\begin{array}{l}\text { React- } \\
\text { ions }\end{array}$ \\
\hline 1 & Zhumki & $\begin{array}{l}53.04 \\
(47.73) \mathrm{bc}\end{array}$ & $\begin{array}{l}26.67 \\
(31.03) \mathrm{ab}\end{array}$ & $\begin{array}{l}45.51 \\
(42.38) \mathrm{a}\end{array}$ & \begin{tabular}{|l|}
71.36 \\
$(58.14) \mathrm{a}$
\end{tabular} & $\begin{array}{l}61.35 \\
(51.73) \mathrm{a}\end{array}$ & HS \\
\hline 2 & ISD-006 & $\begin{array}{l}10.47 \\
(18.65) \mathrm{tu}\end{array}$ & $\begin{array}{l}5.49 \\
(13.36) \mathrm{pqr}\end{array}$ & $\begin{array}{l}13.19 \\
(21.17) t u\end{array}$ & $\begin{array}{l}15.53 \\
(22.64) \mathrm{x}\end{array}$ & $\begin{array}{l}12.67 \\
(20.20) \mathrm{st}\end{array}$ & MR \\
\hline 3 & Laffa M & $\begin{array}{l}13.99 \\
(21.60) \mathrm{rst}\end{array}$ & $\begin{array}{l}7.02 \\
(15.11) \text { n-r } \\
\end{array}$ & $\begin{array}{l}17.71 \\
(24.86) r s\end{array}$ & $\begin{array}{l}29.43 \\
(32.82) \mathrm{t}\end{array}$ & $\begin{array}{l}24.08 \\
(29.35) \mathrm{mno}\end{array}$ & MS \\
\hline 4 & Laffa G & $\begin{array}{l}21.29 \\
(27.36) \text { nop }\end{array}$ & $\begin{array}{ll}10.70 & \\
(18.99) & \text { i-m }\end{array}$ & $\begin{array}{l}23.41 \\
(28.86) 1-q\end{array}$ & \begin{tabular}{|l}
33.38 \\
$(35.24)$ opq
\end{tabular} & $\begin{array}{l}24.17 \\
(29.38) \mathrm{mno}\end{array}$ & $\mathrm{S}$ \\
\hline 5 & Laffa W & $\begin{array}{l}47.69 \\
(43.65) \mathrm{cd}\end{array}$ & $\begin{array}{l}23.89 \\
(29.21) b\end{array}$ & $\begin{array}{l}36.43 \\
(37.09) \mathrm{bcd}\end{array}$ & $\begin{array}{l}53.23 \\
(46.84) \mathrm{ghi}\end{array}$ & $\begin{array}{l}44.63 \\
(41.83) \text { ef }\end{array}$ & HS \\
\hline 6 & Laffa B & $\begin{array}{l}56.02 \\
(48.46) \mathrm{b}\end{array}$ & $\begin{array}{l}27.95 \\
(31.87) \mathrm{ab}\end{array}$ & $\begin{array}{l}39.93 \\
(39.16) \mathrm{b}\end{array}$ & $\begin{array}{l}60.79 \\
(51.30) \mathrm{de}\end{array}$ & $\begin{array}{l}50.93 \\
(45.51) \mathrm{cd}\end{array}$ & HS \\
\hline 7 & Laffa S & $\begin{array}{l}21.27 \\
(27.31) \text { nop }\end{array}$ & $\begin{array}{l}10.81 \\
(19.07) \mathrm{j}-\mathrm{m}\end{array}$ & $\begin{array}{l}23.88 \\
(29.10) k-p\end{array}$ & $\begin{array}{l}36.57 \\
(36.85) \text { no }\end{array}$ & $\begin{array}{l}27.52 \\
(31.21) \mathrm{k}-\mathrm{n}\end{array}$ & S \\
\hline 8 & Laffa BAU & $\begin{array}{l}25.06 \\
(29.97) j-n\end{array}$ & $\begin{array}{l}12.62 \\
(20.74) \text { e-k }\end{array}$ & $\begin{array}{l}25.84 \\
(30.49) j-n \\
\end{array}$ & $\begin{array}{l}37.0 \\
(37.42) \mathrm{mn}\end{array}$ & $\begin{array}{l}22.42 \\
(28.24) \text { nop }\end{array}$ & S \\
\hline 9 & Volanath & $\begin{array}{l}21.39 \\
(27.49) \text { nop }\end{array}$ & \begin{tabular}{|ll}
10.90 & \\
$(19.22) \quad h-m$
\end{tabular} & $\begin{array}{l}22.50 \\
(28.26) \quad m-q\end{array}$ & $\begin{array}{l}32.91 \\
(34.97) \mathrm{pqr}\end{array}$ & $\begin{array}{l}23.31 \\
(28.51) \mathrm{m}-\mathrm{p}\end{array}$ & S \\
\hline 10 & Thamba & $\begin{array}{l}17.99 \\
(24.90) \mathrm{pqr}\end{array}$ & \begin{tabular}{|l|}
8.61 \\
$(16.96) 1-p$
\end{tabular} & \begin{tabular}{|l}
20.87 \\
$(27.03)$ o-r
\end{tabular} & \begin{tabular}{|l|}
30.33 \\
$(33.35) r s t$
\end{tabular} & $\begin{array}{l}20.48 \\
(26.77) \mathrm{opq}\end{array}$ & MS \\
\hline 11 & Dohazari R & $\begin{array}{l}18.46 \\
(25.26) \mathrm{opq}\end{array}$ & $\begin{array}{l}9.24 \\
(17.55) \mathrm{k}-\mathrm{o}\end{array}$ & $\begin{array}{l}19.31 \\
(25.81) \mathrm{qrs}\end{array}$ & $\begin{array}{l}30.98 \\
(33.69) \mathrm{q}-\mathrm{t}\end{array}$ & $\begin{array}{l}24.53 \\
(29.59) 1-\mathrm{o}\end{array}$ & MS \\
\hline 12 & Boruka & $\begin{array}{l}42.33 \\
(40.56) \text { def }\end{array}$ & $\begin{array}{l}18.78 \\
(25.48) \mathrm{c}\end{array}$ & $\begin{array}{l}37.15 \\
(37.50) b c\end{array}$ & $\begin{array}{l}58.01 \\
(49.65) \text { ef }\end{array}$ & $\begin{array}{l}50.90 \\
(45.50) \mathrm{cd}\end{array}$ & HS \\
\hline 13 & Dohazari & $\begin{array}{ll}30.05 \\
(32.88) & h-k \\
\end{array}$ & $\begin{array}{l}15.05 \\
(22.49) \mathrm{c}-\mathrm{i}\end{array}$ & $\begin{array}{l}31.15 \\
(33.62) \mathrm{e}-\mathrm{i}\end{array}$ & $\begin{array}{l}40.57 \\
(39.46) \mathrm{kl}\end{array}$ & $\begin{array}{l}33.32 \\
(35.02) \mathrm{hi}\end{array}$ & HS \\
\hline 14 & Khatkhatia B & $\begin{array}{l}32.60 \\
(34.75) \text { ghi }\end{array}$ & $\begin{array}{ll}16.44 & \\
(23.85) & \mathrm{c}-\mathrm{g}\end{array}$ & $\begin{array}{l}25.55 \\
(30.22) \mathrm{j}-\mathrm{o}\end{array}$ & $\begin{array}{l}39.76 \\
(39.06) \mathrm{lm}\end{array}$ & $\begin{array}{l}31.60 \\
(34.15) h-k\end{array}$ & S \\
\hline 15 & Khatkhatia BAU & $\begin{array}{l}23.42 \\
(28.82) 1-\mathrm{o}\end{array}$ & $\begin{array}{l}11.80 \\
(19.99) \mathrm{h}-1\end{array}$ & \begin{tabular}{|l|}
24.56 \\
$(29.57) \mathrm{k}-\mathrm{o}$
\end{tabular} & $\begin{array}{l}33.94 \\
(35.57) \text { op }\end{array}$ & $\begin{array}{l}27.82 \\
(31.77) j-n\end{array}$ & $\mathrm{~S}$ \\
\hline 16 & Kaikka N & \begin{tabular}{|l|}
20.41 \\
$(26.71)$ nop
\end{tabular} & $\begin{array}{ll}10.25 & \\
(18.57) & j-n\end{array}$ & $\begin{array}{l}21.80 \\
(27.64) \mathrm{m}-\mathrm{r}\end{array}$ & $\begin{array}{l}0.32 \\
(33.38) r s t\end{array}$ & $\begin{array}{l}24.02 \\
(29.32) \mathrm{mno}\end{array}$ & MS \\
\hline 17 & Kaikka G & $\begin{array}{l}24.95 \\
(29.83) j-n\end{array}$ & $\begin{array}{l}12.47 \\
(20.57) 1\end{array}$ & $\begin{array}{l}27.80 \\
(31.75) \mathrm{h}-1\end{array}$ & \begin{tabular}{|l}
39.76 \\
$(39.05) \mathrm{lm}$
\end{tabular} & $\begin{array}{l}32.48 \\
(34.68) h i j\end{array}$ & S \\
\hline
\end{tabular}

Values in a column with same letter(s) do not differ significantly.

Figures in the parentheses are in the arcsin transformed values.

Table2. Continued

\begin{tabular}{|l|c|c|c|c|c|c|c|}
\hline $\begin{array}{l}\text { Sl. } \\
\text { No }\end{array}$ & $\begin{array}{c}\text { Germplasms } \\
\text { /cultivars }\end{array}$ & $\begin{array}{c}\text { \%Leaf } \\
\text { infection }\end{array}$ & $\begin{array}{c}\text { PDI } \\
\text { (Leaf) }\end{array}$ & $\begin{array}{c}\text { \%Flower } \\
\text { infection }\end{array}$ & $\begin{array}{c}\text { \%Fruit } \\
\text { infection }\end{array}$ & $\begin{array}{c}\text { PDI } \\
\text { (fruit) }\end{array}$ & React-ions \\
\hline 18 & $\begin{array}{c}\text { Islampuri } \\
\text { BADC }\end{array}$ & $\begin{array}{c}52.52 \\
(46.43) \mathrm{bc}\end{array}$ & $\begin{array}{c}26.25 \\
(30.77) \mathrm{ab}\end{array}$ & $\begin{array}{c}34.45 \\
(35.81) \mathrm{e}-\mathrm{f}\end{array}$ & $\begin{array}{c}54.66 \\
(47.68) \mathrm{gh}\end{array}$ & $\begin{array}{c}42.49 \\
(40.60) \mathrm{f}\end{array}$ & $\mathrm{HS}$ \\
\hline 19 & Jessore L & $\begin{array}{c}1368 \\
(21.28) \mathrm{st}\end{array}$ & $\begin{array}{c}6.88 \\
(14.39) \mathrm{o}-\mathrm{r}\end{array}$ & $\begin{array}{c}13.14 \\
(20.74) \mathrm{tu}\end{array}$ & $\begin{array}{c}33.77 \\
(21.27) \mathrm{xy}\end{array}$ & $\begin{array}{c}8.55 \\
(16.79) \mathrm{uv}\end{array}$ & $\mathrm{MR}$ \\
\hline 20 & Dharala & 23.22 & 11.75 & 20.97 & 37.00 & 31.65 & $\mathrm{~S}$ \\
\hline
\end{tabular}




\begin{tabular}{|c|c|c|c|c|c|c|c|}
\hline & & (28.71)1-o & (19.97)h-1 & (27.19)n-r & (37.44)mn & (34.20)h-k & \\
\hline 21 & Nayantara & $\begin{array}{c}22.80 \\
(28.43) \mathrm{mp}\end{array}$ & $\begin{array}{c}11.49 \\
(19.84) \mathrm{h}-1\end{array}$ & $\begin{array}{c}24.60 \\
(29.68) \mathrm{k}-\mathrm{o}\end{array}$ & $\begin{array}{c}37.98 \\
(38.01) 1 \mathrm{mn}\end{array}$ & $\begin{array}{c}31.29 \\
(33.97) \mathrm{h}-\mathrm{k}\end{array}$ & $S$ \\
\hline 22 & Uttara & $\begin{array}{c}23.35 \\
(28.76) 1-\mathrm{o}\end{array}$ & $\begin{array}{c}12.12 \\
(20.29) \mathrm{g}-1\end{array}$ & $\begin{array}{c}38.35 \\
(38.29) \mathrm{bc}\end{array}$ & $\begin{array}{c}60.72 \\
(51.27) \mathrm{de}\end{array}$ & $\begin{array}{c}50.80 \\
(45.44) \mathrm{cd}\end{array}$ & HS \\
\hline 23 & Kazla & $\begin{array}{c}45.29 \\
(42.27) \mathrm{dc}\end{array}$ & $\begin{array}{c}24.35 \\
(29.43) \mathrm{b}\end{array}$ & $\begin{array}{c}46.68 \\
(43.07) \mathrm{a}\end{array}$ & $\begin{array}{c}69.87 \\
(57.00) \mathrm{ab}\end{array}$ & $\begin{array}{c}59.71 \\
(50.69) \mathrm{a}\end{array}$ & HS \\
\hline 24 & Singnath C & $\begin{array}{c}23.87 \\
(29.15) \mathrm{k}-\mathrm{n}\end{array}$ & $\begin{array}{c}11.95 \\
(20.14) \mathrm{g}-1\end{array}$ & $\begin{array}{c}17.87 \\
(24.81) \mathrm{rs}\end{array}$ & $\begin{array}{c}23.29 \\
(28.81) \mathrm{v}\end{array}$ & $\begin{array}{c}19.04 \\
(25.82) \mathrm{pq}\end{array}$ & MS \\
\hline 25 & BL-118 & $\begin{array}{c}40.10 \\
(39.27) \mathrm{ef}\end{array}$ & $\begin{array}{c}18.58 \\
(25.01) \mathrm{cd}\end{array}$ & $\begin{array}{c}21.04 \\
(27.27) n-r\end{array}$ & $\begin{array}{c}37.44 \\
(37.70) 1 \mathrm{mn}\end{array}$ & $\begin{array}{c}30.04 \\
(33.20) \mathrm{ijk}\end{array}$ & $S$ \\
\hline 26 & Dundul & $\begin{array}{c}11.90 \\
(19.58) \mathrm{stu}\end{array}$ & $\begin{array}{c}6.01 \\
(13.74) \mathrm{pqr}\end{array}$ & $\begin{array}{c}12.92 \\
(20.52) \mathrm{u}\end{array}$ & $\begin{array}{c}18.00 \\
(24.71) \mathrm{w}\end{array}$ & $\begin{array}{c}12.51 \\
(20.44) \mathrm{st}\end{array}$ & MR \\
\hline 27 & Botli begun & $\begin{array}{c}33.10 \\
(35.07) \mathrm{ghi}\end{array}$ & $\begin{array}{c}16.70 \\
(24.08) \mathrm{c}-\mathrm{f}\end{array}$ & $\begin{array}{c}19.60 \\
(26.22) \mathrm{e}-\mathrm{r}\end{array}$ & $\begin{array}{c}31.85 \\
(34.32) \mathrm{p}-\mathrm{t}\end{array}$ & $\begin{array}{c}22.60 \\
\text { (27.88)op }\end{array}$ & $S$ \\
\hline 28 & $\begin{array}{c}\text { Marich begun } \\
\mathrm{S}\end{array}$ & $\begin{array}{c}8.42 \\
(16.68) \mathrm{u}\end{array}$ & $\begin{array}{c}4.22 \\
(11.70) \mathrm{r}\end{array}$ & $\begin{array}{c}8.65 \\
(16.95) \mathrm{v}\end{array}$ & $\begin{array}{c}12.86 \\
(20.87) \mathrm{y}\end{array}$ & $\begin{array}{c}10.05 \\
(18.24) \mathrm{tu}\end{array}$ & MR \\
\hline 29 & $\begin{array}{c}\text { Marich begun } \\
\mathrm{E}\end{array}$ & $\begin{array}{c}8.76 \\
(17.07) \mathrm{u}\end{array}$ & $\begin{array}{c}4.31 \\
(11.87) \mathrm{r}\end{array}$ & $\begin{array}{c}12.45 \\
(20.62) \mathrm{tu}\end{array}$ & $\begin{array}{c}21.38 \\
(27.49) \mathrm{v}\end{array}$ & $\begin{array}{c}17.27 \\
(24.49) \mathrm{ur}\end{array}$ & MR \\
\hline 30 & $\begin{array}{c}\text { Marich begun } \\
\text { L }\end{array}$ & $\begin{array}{c}9.08 \\
(17.40) \mathrm{u}\end{array}$ & $\begin{array}{c}4.53 \\
(12.17) \mathrm{qr}\end{array}$ & $\begin{array}{c}15.95 \\
(23.50) \mathrm{stu}\end{array}$ & $\begin{array}{c}29.99 \\
(33.16) \mathrm{st}\end{array}$ & $\begin{array}{c}24.55 \\
(29.63) 1-0\end{array}$ & MR \\
\hline 31 & Katabegun Ws & $\begin{array}{c}0.00 \\
(0.37) \mathrm{v}\end{array}$ & $\begin{array}{c}0.00 \\
(0.37) \mathrm{s}\end{array}$ & $\begin{array}{c}0.00 \\
(0.37) \mathrm{w}\end{array}$ & $\begin{array}{c}0.00 \\
(0.37) /\end{array}$ & $\begin{array}{c}0.00 \\
(0.37) \mathrm{w}\end{array}$ & $\mathrm{R}$ \\
\hline 32 & Bijoy & $\begin{array}{c}25.15 \\
(29.97) \mathrm{j}-\mathrm{n}\end{array}$ & $\begin{array}{c}12.50 \\
(20.60) \mathrm{f}-1\end{array}$ & $\begin{array}{c}24.29 \\
(29.41) \mathrm{k}-\mathrm{p}\end{array}$ & $\begin{array}{c}33.10 \\
(35.08) \mathrm{pqr}\end{array}$ & $\begin{array}{c}23.91 \\
(28.71) \mathrm{m}-\mathrm{p}\end{array}$ & $S$ \\
\hline 33 & Amjuri & $\begin{array}{c}14.75 \\
(22.43) \mathrm{qrs}\end{array}$ & $\begin{array}{c}7.49 \\
(15.66) \mathrm{m}-\mathrm{q}\end{array}$ & $\begin{array}{c}17.97 \\
(24.97) \mathrm{rs}\end{array}$ & $\begin{array}{c}26.80 \\
(31.12) \mathrm{u}\end{array}$ & $\begin{array}{c}22.00 \\
\text { (27.88)op }\end{array}$ & MS \\
\hline 34 & EG-190 & $\begin{array}{c}52.71 \\
(46.07) \mathrm{bc}\end{array}$ & $\begin{array}{c}28.64 \\
(32.33) \mathrm{ab}\end{array}$ & $\begin{array}{c}39.76 \\
(39.07) \mathrm{b}\end{array}$ & $\begin{array}{c}67.88 \\
(55.50) \mathrm{b}\end{array}$ & $\begin{array}{c}58.03 \\
(49.62) \mathrm{ab}\end{array}$ & HS \\
\hline 35 & China & $\begin{array}{c}52.41 \\
(46.37) \mathrm{bc}\end{array}$ & $\begin{array}{c}28.22 \\
(21.97) \mathrm{ab}\end{array}$ & $\begin{array}{c}35.44 \\
(36.50) b-e\end{array}$ & $\begin{array}{c}55.85 \\
(48.35) f g \\
\end{array}$ & $\begin{array}{c}47.89 \\
\text { (43.76)de }\end{array}$ & HS \\
\hline 36 & Wild & $\begin{array}{c}0.00 \\
(0.37) \mathrm{v}\end{array}$ & $\begin{array}{c}0.00 \\
(0.37) \mathrm{s}\end{array}$ & $0.00(0.37) \mathrm{w}$ & $\begin{array}{c}0.00 \\
(0.37) /\end{array}$ & $\begin{array}{c}0.00 \\
(0.37) \mathrm{w}\end{array}$ & $\mathrm{R}$ \\
\hline
\end{tabular}

Values in a column with same letter(s) do not differ significantly.

Figures in the parentheses are in the arcsin transformed values.

Table2. Continued

\begin{tabular}{|l|l|l|l|l|l|l|l|}
\hline $\begin{array}{l}\text { Sl. } \\
\text { No }\end{array}$ & $\begin{array}{l}\text { Germplasms } \\
\text { /cultivars }\end{array}$ & $\begin{array}{l}\text { \% Leaf } \\
\text { infection }\end{array}$ & $\begin{array}{l}\text { PDI } \\
\text { (Leaf) }\end{array}$ & $\begin{array}{l}\% \text { Flower } \\
\text { infection }\end{array}$ & $\begin{array}{l}\text { \% Fruit } \\
\text { infection }\end{array}$ & $\begin{array}{l}\text { PDI } \\
\text { (fruit })\end{array}$ & React-ions \\
\hline 37 & Rupgonj L & $\begin{array}{l}61.74 \\
(51.82) \mathrm{a}\end{array}$ & $\begin{array}{l}30.85 \\
(33.70) \mathrm{a}\end{array}$ & $\begin{array}{l}38.27 \\
(38.18) \mathrm{bc}\end{array}$ & $\begin{array}{l}63.06 \\
(52.64) \mathrm{cd}\end{array}$ & $\begin{array}{l}52.63 \\
(46.49) \mathrm{cd}\end{array}$ & HS \\
\hline 38 & Ishurdi WS & $\begin{array}{l}8.25 \\
(16.64) \mathrm{u}\end{array}$ & $\begin{array}{l}4.15 \\
(11.71) \mathrm{r}\end{array}$ & $\begin{array}{l}6.07 \\
(14.16) \mathrm{v}\end{array}$ & $\begin{array}{l}4.48 \\
(16.87) \ldots . .\end{array}$ & $\begin{array}{l}6.66 \\
(14,89) \mathrm{v}\end{array}$ & MR \\
\hline 39 & Ishurdi BS & $\begin{array}{l}8.40 \\
(16.79) \mathrm{u}\end{array}$ & $\begin{array}{l}7.12 \\
(14.12) \mathrm{o}-\mathrm{r}\end{array}$ & $\begin{array}{l}6.63 \\
(14.80) \mathrm{v}\end{array}$ & $\begin{array}{l}10.32 \\
(18.70) \mathrm{z}\end{array}$ & $\begin{array}{l}8.10 \\
(16.48) \mathrm{uv}\end{array}$ & MR \\
\hline 40 & Putabegun & $\begin{array}{l}29.41 \\
(32.77) \mathrm{h}-\mathrm{k}\end{array}$ & $\begin{array}{l}14.74 \\
(22.52) \mathrm{c}-\mathrm{i}\end{array}$ & $\begin{array}{l}26.26 \\
(30.76) \mathrm{j}-\mathrm{m}\end{array}$ & $\begin{array}{l}45.05 \\
(42.13) \mathrm{j}\end{array}$ & $\begin{array}{l}36.61 \\
(37.19) \mathrm{gh}\end{array}$ & $\mathrm{S}$ \\
\hline 41 & Longla L & $\begin{array}{l}38.22 \\
(38.12) \mathrm{fg}\end{array}$ & $\begin{array}{l}18.02 \\
(25.09) \mathrm{cd}\end{array}$ & $\begin{array}{l}29.83 \\
(33.04) \mathrm{f}-\mathrm{j}\end{array}$ & $\begin{array}{l}50.38 \\
(45.20) \mathrm{i}\end{array}$ & $\begin{array}{l}40.41 \\
(39.44) \mathrm{fg}\end{array}$ & $\mathrm{S}$ \\
\hline 42 & Shingnath S & $\begin{array}{l}21.12 \\
(27.30) \mathrm{nop}\end{array}$ & $\begin{array}{l}10.71 \\
(19.06) \mathrm{i}-\mathrm{n}\end{array}$ & $\begin{array}{l}21.20 \\
(27.36) \mathrm{n}-\mathrm{r}\end{array}$ & $\begin{array}{l}36.10 \\
(36.90) \mathrm{no}\end{array}$ & $\begin{array}{l}32.15 \\
(34.51) \mathrm{hij}\end{array}$ & $\mathrm{S}$ \\
\hline 43 & Longla T & $\begin{array}{l}36.84 \\
(37.35) \mathrm{fg}\end{array}$ & $\begin{array}{l}18.50 \\
(25.45) \mathrm{c}\end{array}$ & $\begin{array}{l}28.39 \\
(32.17) \mathrm{j}-\mathrm{k}\end{array}$ & $\begin{array}{l}52.81 \\
(46.59) \mathrm{hi}\end{array}$ & $\begin{array}{l}42.32 \\
(40.56) \mathrm{f}\end{array}$ & $\mathrm{S}$ \\
\hline 44 & Islampuri I & $\begin{array}{l}62.21 \\
(52.06) \mathrm{a}\end{array}$ & $\begin{array}{l}31.19 \\
(33.93) \mathrm{a}\end{array}$ & $\begin{array}{l}37.78 \\
(37.36) \mathrm{bcd}\end{array}$ & $\begin{array}{l}62.54 \\
(52.24) \mathrm{cd}\end{array}$ & $\begin{array}{l}52.45 \\
(46.38) \mathrm{cd}\end{array}$ & HS \\
\hline 45 & Thapra & $\begin{array}{l}29.95 \\
(33.16) \mathrm{hij}\end{array}$ & $\begin{array}{l}15.31 \\
(23.01) \mathrm{c}-\mathrm{h}\end{array}$ & $\begin{array}{l}33.22 \\
(35.17) \mathrm{c}-\mathrm{r}\end{array}$ & $\begin{array}{l}43.34 \\
(41.15) \mathrm{jk}\end{array}$ & $\begin{array}{l}33.20 \\
(35.17) \mathrm{hi}\end{array}$ & $\mathrm{S}$ \\
\hline 46 & Mireshari & $\begin{array}{l}27.16 \\
(31.38) \mathrm{i}-\mathrm{m}\end{array}$ & $\begin{array}{l}13.63 \\
(21.64) \mathrm{d}-\mathrm{j}\end{array}$ & $\begin{array}{l}31.66 \\
(34.21) \mathrm{d}-\mathrm{h}\end{array}$ & $\begin{array}{l}43.15 \\
(41.05) \mathrm{jk}\end{array}$ & $\begin{array}{l}33.10 \\
(35.10) \mathrm{hi}\end{array}$ & $\mathrm{S}$ \\
\hline
\end{tabular}




\begin{tabular}{|c|c|c|c|c|c|c|c|}
\hline 47 & Menter & \begin{tabular}{|l|}
28.25 \\
$(32.08) h-m$
\end{tabular} & $\begin{array}{l}14.22 \\
(22.13) c-j\end{array}$ & \begin{tabular}{|l}
46.29 \\
$(42.85) a$
\end{tabular} & \begin{tabular}{|l}
65.05 \\
$(53.83) c$
\end{tabular} & $\begin{array}{l}53.60 \\
(47.06) b c\end{array}$ & HR \\
\hline 48 & Salta & $\begin{array}{l}14.52 \\
(22.36) q r s\end{array}$ & $\begin{array}{l}7.37 \\
(15.72) \mathrm{m}-\mathrm{q}\end{array}$ & $\begin{array}{l}16.14 \\
(23.66) \mathrm{st}\end{array}$ & \begin{tabular}{|l|}
19.07 \\
$(25.87) \mathrm{w}$
\end{tabular} & $\begin{array}{l}15.10 \\
(22.83) \mathrm{rs}\end{array}$ & MR \\
\hline 49 & Iribegun & $\begin{array}{l}24.33 \\
(29.52) j . n\end{array}$ & $\begin{array}{l}11.77 \\
(20.01) \mathrm{h}-1\end{array}$ & $\begin{array}{l}24.75 \\
(29.80) \mathrm{k}-\mathrm{o}\end{array}$ & $\begin{array}{l}32.43 \\
(34.69) p-s\end{array}$ & \begin{tabular}{|l|}
24.27 \\
$(29.49) 1-\mathrm{o}$
\end{tabular} & MS \\
\hline 50 & Eyered & $\begin{array}{l}29.00 \\
(32.56) \mathrm{h}-1\end{array}$ & $\begin{array}{l}14.47 \\
(22.34) c-j\end{array}$ & $\begin{array}{l}34.85 \\
(36.15) b-f\end{array}$ & $\begin{array}{l}44.36 \\
(41.74) \mathrm{j}\end{array}$ & $\begin{array}{l}35.73 \\
(36.68) \mathrm{gh}\end{array}$ & $S$ \\
\hline 51 & Khatkhatia R & $\begin{array}{l}34.02 \\
(35.65) \mathrm{gh}\end{array}$ & $\begin{array}{l}17.16 \\
(24.44) \text { cde }\end{array}$ & $\begin{array}{l}39.79 \\
(39.69) b\end{array}$ & \begin{tabular}{|l|}
43.33 \\
$(41.14) \mathrm{jk}$
\end{tabular} & $\begin{array}{l}33.35 \\
(35.24) \mathrm{hi}\end{array}$ & $S$ \\
\hline 52 & Deem begun & $\begin{array}{l}27.51 \\
(31.61) 1-\mathrm{m}\end{array}$ & $\begin{array}{l}13.82 \\
(21.80) c-j\end{array}$ & $\begin{array}{l}25.20 \\
(30.10(\mathrm{j}-\mathrm{o})\end{array}$ & $\begin{array}{l}38.60 \\
(38.39) \mathrm{lmn}\end{array}$ & \begin{tabular}{|l}
28.98 \\
$(32.54) \mathrm{g}-1$
\end{tabular} & MS \\
\hline 53 & Comilla L & $\begin{array}{l}29.49 \\
(32.86) \mathrm{h}-\mathrm{k}\end{array}$ & $\begin{array}{l}14.92 \\
(22.70) \mathrm{c}-\mathrm{i}\end{array}$ & $\begin{array}{l}33.69 \\
(35.46) c-f\end{array}$ & $\begin{array}{l}42.71 \\
(40.78) \mathrm{jk}\end{array}$ & $\begin{array}{l}32.39 \\
(34.65) h i j\end{array}$ & $S$ \\
\hline
\end{tabular}

Values in a column with same letter(s) do not differ significantly.

Figures in the parentheses are in the arcsin transformed values.

\section{DISCUSSION}

Out of 53 eggplant germplasms, Katabegun WS and a wild accession were found resistant against $P$. vexans through consecutive 3 years screening observation. The present findings are in line with the previous studies in Bangladesh (Islam 2005; Sharker, 2004 and Meah, 2003. Meah et al. (1998) reported Katabegun as tolerant against Phomopsis blight and fruit rot of eggplant. Sharker (2004) noted Katabegun WS as resistant against $P$. vexans. Meah (2003) observed Katabegun WS and Katabegun wild as resistant against P.vexans. Similar studies in India, USA and Porto Rico and Philippine revealed the existence of differential response among eggplant varieties to infection by $P$. vexans under inoculation test (Khodke, 1990, Quaiser and Ahmad, 1987). Studies in India indicated varieties like Pusa dwarf and Annamalai as moderately susceptible and some wild Solanum spp. as highly resistant to immune (Quaiser and Ahmad, 1987). In another study, varieties Pusa dwarf green, Pusha purple round, Pusha purple long, black beauty and thorny monjory gota found resistant (Khodke, 1990). As per report of Edgarten and Moreland (1921) varieties Black beauty, Mammoth purple and Florida high bush were less susceptible in Louisiana, USA. A Brazilian variety Gilo, a tall shrub with small red fruits was reported immune (Howard and Desrosiers, 1943). These reports are not directly related to the result of the present investigation, as the varieties used in the investigation were different. However, report of Howard and Desrosiers (1943) stating the existence of high degree of resistance in the Indian Pegan and Bengal strains can be judiciously related to the findings of the present investigation. Hence a variable disease reaction was observed in the present study which will be useful for development of disease resistant eggplant cultivars in Bangladesh.

\section{CONCLUSION}

Altogether 53 germplasms were collected from different eggplant pockets of the country which were showed differential reactions against Phomopsis vexans. Among the 53 accessions where 13 cultivars were found highly susceptible, 21 cultivars susceptible, 8 cultivars moderately susceptible and 9 showed moderately resistant while cultivar Katabegun-WS and wild accession were resistant. Relatedness of eggplant against phomopsis fruit rot disease reaction could be potential information for developing the disease resistant cultivar of its.

\section{ACKNOWLEDGEMENTS}

The authors thankfully acknowledge Bangladesh Agricultural Research Institute, Gazipur to provide financial support and logistic support. Special thanks to Dr. Firoza Khatun, Chief Scientific Officer and Head Plant Pathology Division, BARI for her fruitful discussions. Thanks go to Most. Shainur Akter (Scientific Assistant) for her sincere assistance in this research work.

\section{REFERENCES}

[1] Ahmad Q. 1987. Sources of resistance in brinjal to Phomopsis fruit rot. Indian Phytopathol. 40(1): 89.

[2] Annonymous.1988. Fal, Shaksabji O Masla Jatio Fasal. Department of Agriculture Extension, Khamarbari, Dhaka, Bangladesh. p.26. 
[3] Anonymous. 2012. Fertilizer Recommendation Guide (FRG), Bangladesh Agricultural Research Council, Farmgate, Dhaka. Pp.70-71.

[4] Anonymous. 2019. Statistical Yearbook of Bangladesh. Bangladesh Bureau of Statistics. Ministry of Planning, Government of the People's Republic of Bangladesh. Dhaka, Bangladesh. pp. 249-290.

[5] Khan NU.1999. Occurrence, seed-borne nature and management of Phomopsis blight of eggplant. An M.S. thesis submitted to the Department of Plant Pathology. BAU, Mymensingh.pp.32-66.

[6] Das BH. 1998. Studies on Phomopsis fruit rot of brinjal. An M.S. thesis submitted to the Department of Plant Pathology. BAU, Mymensingh.pp.29-64.

[7] Dashgupta MK. 1988. Principles of Plant Pathology. Allied Publisher Private Limited. New Delhi, India. 700 pp.

[8] Edgarten CW and Moreland CC. 1921. Eggplant blight. Rev. appl. Mycol. 1: 197.

[9] Fakir GA. 1983. Root and stem rot of brinjal caused by Phomopsis vexans. Proceedings of 8th Bangladesh Sci. Con. Section 1 pp.67-68.

[10] Howard FL and Desrosiers R. 1943. Studies on the resistance of eggplant varieties to phomopsis blight. Rev. Appl. Mycol. 22: 194.

[11] Islam SK, Sitansu P and Pan S. 1990. Efficacy of humidity and temperature on Phomopsis fruit rot of brinjal (Solanum melongena). Environment and Ecology 8(4): 1309-1310.

[12] Islam MR. 2005. An integrated approach for management of Phomopsis blight and fruit rot of eggplant. A Ph.D. thesis, Department of Plant Pathology, Bangladesh Agricultural University, Mymensingh-2202. pp.166.

[13] Kalda TS, Swarup V and Choudhury B. 1977. Resistance to Phomopsis blight in eggplant. Vegetable Sci. India. 4(2): 90-101.

[14] Khodke PS. 1990. Host range and varietal resistance to stem blight (Phomopsis vexans) of brinjal. Indian Phytopath. 43(2): 315.

[15] Kumar A, Aulakh KS and Grewal RK.1986.Incidence of fruit rot of brinjal in Punjab. Indian Phytopath. 39(3): 482.

[16] Meah MB. 2003. Integrated Management of Eggplant Cultivation-I. USDA-Bangladesh Collaborative Research Project, IPM Lab, Department of Plant Pathology, Bangladesh Agricultural University, Mymensingh-2202, Bangladesh. Pp. 30.

[17] Meah MB, Das BH, Siddiqua MK and Nasir M. 1998. Screening Brinjal varieties and fungicide against Phomopsis rot. Bangladesh J. Bot. 19(1): 5-20.

[18] Quaiser A and Ahmed Q. 1987. Sources of resistance in brinjal to phomopsis fruit rot. Indian Phytopath. 40(1):98-99.

[19] [Sarker MFK. 2004. Morphological and anatomical features of eggplant contributing to resistance to Phomopsis blight and fruit rot, foot rot and shoot and fruit borer. An M. S. thesis. Department of Plant Pathology, Bangladesh Agricultural University, Mymensingh. pp. 35-45.

Citation: M.M. Islam, M.I. Faruk, M.S. Rahman, K.E. Jahan, M. Asaduzzaman, Screening of Eggplant Germplasms against Phomopsis Blight and Fruit Rot Caused by Phomopsis Vexans. International Journal of Research Studies in Biosciences. 2020; 8(7): 28-34. DOI: https://doi.org/10.20431/2349-0365.0807005.

Copyright: (C) 2020 Authors. This is an open-access article distributed under the terms of the Creative Commons Attribution License, which permits unrestricted use, distribution, and reproduction in any medium, provided the original author and source are credited. 